\title{
Black-Box Modeling of EMI Filters for Frequency and Time-Domain Simulations
}

\author{
Simone Negri, Member, IEEE, Giordano Spadacini, Senior Member, IEEE, Flavia Grassi, Senior \\ Member, IEEE, and Sergio A. Pignari, Fellow, IEEE
}

\begin{abstract}
A procedure for the derivation of a black-box model of electromagnetic interference (EMI) filters is proposed and discussed. The modeling approach is assessed by resorting to a real EMI filter. The equivalent circuit of the filter is directly obtained from a rational approximation of the scattering-parameter matrix measured at the filter ports. Therefore, the modeling procedure does not require any information on the internal structure of the filter (e.g., components, electrical/magnetic properties of the involved materials, connections leads, etc.). The circuit model is compatible with SPICE solvers and can be used for the prediction of conducted emissions in both the frequency and time domain. Specifically, the proposed modeling approach allows time-domain simulation and performance analysis of EMI filters in combination with power-electronic equipment (i.e. non-linear, time-variant circuits).
\end{abstract}

Index Terms-Conducted emission (CE), electromagnetic interference (EMI), EMI filters, power electronics.

\section{INTRODUCTION}

$\mathrm{I}_{\mathrm{N}}^{\mathrm{N}}$ $\mathrm{N}$ recent years, the availability of powerful electromagnetic and circuit simulation tools had a profound impact on electromagnetic compatibility activities in industry. Previous trial-and-error approaches based on iterative experiments are increasingly being replaced by simulation for virtual prototyping, thus reducing development costs and time to market. In this respect, the prediction of conducted emissions (CE) in power-electronic equipment has made great strides.

The pioneering works [1], [2] exemplified how the mechanism of CE generation and propagation can be modeled in two distinctive domains of interest: time and frequency. Frequency-domain modeling is appealing for direct connection to EMC standards and regulations (emission limits, resolution bandwidth, etc.), whereas time-domain simulation allows for modeling non-linear and/or time-variant effects in electronic equipment.

For effective characterization of power-electronic equipment as $\mathrm{CE}$ sources in the frequency domain, experimental methods to identify approximate, linear behavioral models composed of frequency-dependent impedances and sources were presented in [3], [4]. Together with circuit representations of lineimpedance stabilization networks (LISNs), energy sources, lumped or distributed-parameter models of cables, these frequency-domain techniques proved to be effective for $\mathrm{CE}$ analysis in complex systems [5], [6].

Manuscript received Apr. 26, 2021, revised July 2, 2021, accepted July 30 , 2021.

S. Negri, G. Spadacini, F. Grassi, and S. A. Pignari are with Politecnico di Milano, Dept. of Electronics, Information and Bioengineering, 20133, Milan,
Circuit simulation in the time domain (e.g., in the SPICE environment) is more challenging than frequency-domain analysis, as it involves detailed, component-level representation of power converters (including valves and their switching activity) [7]. Moreover, the inclusion of bus-bar capacitances and other parasitic parameters is fundamental in time-domain circuit models [8], even though estimation of those parameters is subject to uncertainty. Impedance measurement [9] or 3D electromagnetic solvers can be used to extract such parasitics [10].

From an industrial perspective, simulation of $\mathrm{CE}$ demonstrates great potential when targeted to design or assessment of the optimal EMI filter [5]. Unfortunately, as far as the evaluation of commercial EMI filters is concerned, the input information required to accomplish that task is hardly made available by the manufacturers. Generally, descriptions provided in data sheets are limited to a) circuit topology with nominal values of the circuit components; b) plots of the insertion loss (IL) defined and experimentally determined according to standard CISPR 17 as measures of the attenuation of the common-mode (CM) and the differential-mode (DM) EMI [11].

Indeed, any circuit representation of EMI filters not taking into account parasitic phenomena is scarcely useful for filter performance prediction, since above a few $\mathrm{kHz}$ the filter frequency response is generally dominated by the nonideal behavior of capacitors and CM chokes, as well as by inductive/capacitive coupling among neighboring components [12]. The idea to augment the circuit model by adding extra elements to model parasitic effects (e.g., capacitances and inductances) was proposed in [13], however, it would hardly be feasible for complex filters (e.g., high-power filters not mounted on printed circuit boards), without access to single components/stages, and seeking accuracy above few tens of MHz. Full-wave electromagnetic simulation of the internal structure is theoretically a viable option but requires comprehensive knowledge of the filter (i.e., internal layout, geometry and electrical parts, magnetic properties of ferrites, etc.) [14]. Unfortunately, that technical information is often undisclosed.

On the other hand, the IL is not useful to infer filter models and suffers from inherent limitations even as a figure of merit. Namely, the IL is defined in setups involving specific source

Italy (e-mail: \{simone.negri, giordano.spadacini, flavia.grassi, sergio.pignari\} (a)polimi.it). 
and load resistance values $(50 \Omega)$, typically not representative of the real, frequency-dependent impedances of the systems in which the EMI filters are mounted and operated. Moreover, linearity is an intrinsic assumption of the frequency domain where the concept of IL is defined. Therefore, the impact of nonlinearity and time-variance of electronic systems on the EMI filter performance cannot be accounted for.

To offer a robust, industrial solution for the inclusion of EMI filters in CE simulation environments, this paper presents a procedure to derive an equivalent circuit model of the EMI filter. Specifically, a "black-box" model is proposed, whose parameters are extracted from the scattering-parameter (Sparameter) matrix measured at the filter ports via a Vector Network Analyzer (VNA) [15], thus not requiring disclosure of any information about the inner structure (components, properties of materials, etc.). The entries of the measured Sparameter matrix are approximated by rational pole-residue expansions through the Vector Fitting (VF) algorithm [16]-[18] and passivity-enforcement techniques [19], [20]. In order to maximize accuracy, the equivalent circuit is directly synthesized from the poles and residues of the S-parameter matrix without any pre-processing (e.g., without any conversion from the S-parameter to the admittance or impedance matrix representation). The proposed black-box model can be used in any CE modeling framework, both in frequency and time domain. In particular, it is compatible with SPICE solvers and gives full potential for time-domain circuit simulation of power-electronic converters.

The paper is organized as follows. In Section II, the three steps of the modeling procedure (S-parameter measurement, rational approximation, and circuit synthesis) are outlined in general. In Section III, a specific EMI filter is presented to exemplify the proposed procedure. In Section IV, the obtained equivalent SPICE circuit is used for frequency-domain simulation of the CISPR-17 IL and validated versus measurements up to $100 \mathrm{MHz}$. Section V presents SPICE timedomain simulations of a buck-converter. Finally, Section VI draws concluding remarks.

\section{Modeling Procedure}

\section{A. Measurement of the S-parameter Matrix}

In the first step of the proposed procedure, the EMI filter is treated as a multiport network, whose frequency response is characterized by S-parameters measurement carried out by a VNA [15]. Linearity is the basic assumption behind this approach, so saturation effects (e.g., due to dc or low frequency $\mathrm{CM}$ currents in CM chokes) are excluded likewise for IL measurements in CISPR 17 [11].

The number of electric ports $N_{p}$ depends on filter type and equals twice the number of system wires, according to Table 1. Each port is defined between a wire terminal and a commonground reference represented by the metallic chassis of the filter. Without loss of generality, numbering starts at the "line" side and ends at the "load" side of the filter, as exemplified in Fig. 1 for case $N_{p}=4$.

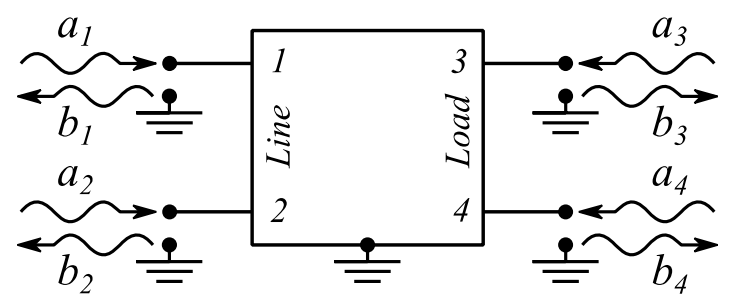

Fig. 1. Definition of ports for S-parameter measurement (dc or single-phaseac EMI filter)

TABLE I

EMI-FILTER TYPE AND NUMBER OF PORTS

\begin{tabular}{ccc}
\hline \hline Type & Wires & $N_{p}$ \\
\hline dc &,+- & 4 \\
single-phase ac & P, N & 4 \\
three-phase ac without neutral & P1, P2, P3 & 6 \\
three-phase ac with neutral & P1, P2, P3, N & 8 \\
\hline \hline
\end{tabular}

\section{B. Rational Approximation of the S-parameter Matrix}

The second step of the proposed procedure consists in the rational approximation of the measured $N_{p} \times N_{p}$ S-parameter matrix $\mathbf{S}$ to fit a pole-residue representation in the form:

$$
\mathbf{S}(s)=\sum_{k=1}^{n} \frac{1}{s-p_{k}} \mathbf{R}_{k}+\mathbf{D}+s \mathbf{E}
$$

where $s=j \omega$ is the complex frequency, $p_{k}$ with $k=1, \ldots, n$ are a common set of $n$ poles, $\mathbf{R}_{k}$ are residue matrices associated to each pole, matrices $\mathbf{D}$ and $\mathbf{E}$ set the low-frequency asymptotic behavior.

The well-known VF algorithm [18] is a robust iterative approach based on least-squares optimization which is best suited to accomplish this numerical task with computational efficiency and high accuracy. Since the obtained rational approximation (1) may lose the physical property of passivity, VF must be complemented by passivity-check and enforcement techniques [19], [20]. Such macromodeling methods were subject of extensive research in recent years, and a summary is out of the scope of this paper. For their principles, the reader is referred to a comprehensive literature source [21] and references therein. Conversely, implementation aspects relevant to the proposed application will be thoroughly discussed in Section III.

\section{Synthesis of an Equivalent Circuit}

Once a passive rational approximation (1) of the S-parameter matrix is obtained, the last step consists in the derivation of a SPICE-compatible equivalent circuit of the EMI-filter at external ports. To this aim, several methodologies are available in the literature [21].

A straightforward approach would be based on properties of the symmetrical admittance matrix $\mathbf{Y}$ of a passive, reciprocal multiport, from which the elements of the branch-admittance matrix $\mathbf{Y}_{B}$ are defined by 


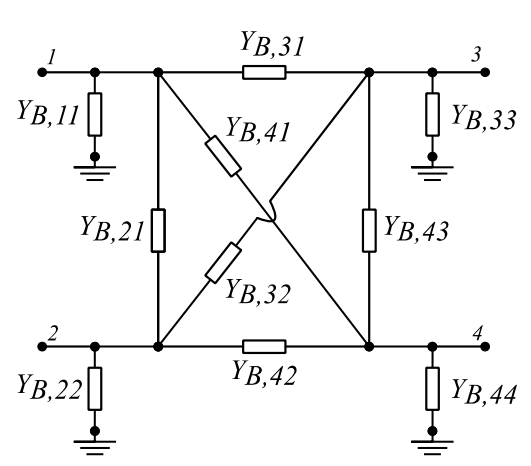

Fig. 2. Equivalent circuit derived from the branch-admittance matrix $\mathbf{Y}_{B}$ (case $\left.N_{\mathrm{p}}=4\right)$

$$
\begin{gathered}
Y_{B, i i}=\sum_{j=1}^{N_{p}} Y_{i j} \text { for } i=1, \ldots, N_{p} \\
Y_{B, i j}=-Y_{i j} \quad \text { for } i, j=1, \ldots, N_{p} \text { and } i \neq j
\end{gathered}
$$

and are directly related to an equivalent circuit composed of admittances connected between nodes in correspondence to the $N_{p}$ ports and ground. In particular, $Y_{B, i i}$ in (2) are admittances connected between node $i$ and the common reference ground, whereas $Y_{B, i j}=Y_{B, j i}$ in (3) are admittances connected between nodes $i$ and $j$. As an example, for the 4-port EMI filter in Fig. 1, the equivalent circuit is sketched in Fig. 2. Since each admittance is expanded by a rational approximation in terms of poles and residues, it can be easily synthesized by resistors, inductors, capacitors and translated into a SPICE netlist [22].

However, this simple approach is not recommended for EMI filters, since it would require the preliminary transformation of the measured matrix $\mathbf{S}$ into the admittance matrix $\mathbf{Y}$ and VF of Y. The measurement errors would propagate through conversion equations and corrupt the derived admittances to a critical extent, implying (a) the need to greatly increase the number of poles $n$ in the VF approximation; (b) difficult or impossible enforcement of passivity. The large magnitude imbalance (e.g., high line-to-ground admittances vs small lineto-line admittances) is another reason why the use of the circuit topology in Fig. 2 favors numerical errors.

Consequently, a circuit model must be synthesized directly from the VF approximation (1) of the measured S-parameters. Unlike other literature approaches [21], the proposed solution aims at preserving the rationale of the branch-admittance synthesis with the introduction of a minimum number of dependent sources. This is done by observing a formal analogy between the admittance and S-parameter representations

$$
\mathbf{I}=\mathbf{Y V} \Leftrightarrow \mathbf{B}=\mathbf{S A}
$$

where $\mathbf{V}, \mathbf{I}$ are vectors of port voltages and currents, respectively, $\mathbf{A}$ and $\mathbf{B}$ are vectors of incident and reflected waves, respectively. Hence, the S-parameter matrix $\mathbf{S}$ can be treated as an analogous admittance matrix $\mathbf{Y}$ in a circuit, provided that the obtained multiport is fed by analogous voltages and currents which are numerically equal to the incident and reflected waves, respectively.

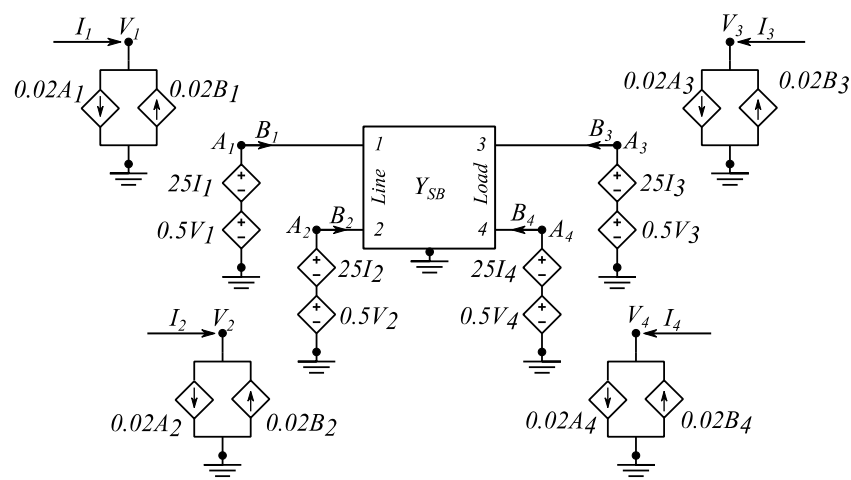

Fig. 3. Equivalent circuit directly obtained from the scattering-parameter matrix $\mathbf{S}$

This consideration suggests the equivalent circuit depicted in Fig. 3 (example for $N_{p}=4$ ), where four controlled sources at the $i$-th port are used to interface the physical voltage $V_{i}$ and current $I_{i}$ with the analogous voltage $A_{i}$ and current $B_{i}$ according to the linear transformation

$$
\begin{aligned}
& A_{i}=\frac{1}{2}\left(V_{i}+R_{0} I_{i}\right) \\
& B_{i}=\frac{1}{2}\left(V_{i}-R_{0} I_{i}\right)
\end{aligned}
$$

where $R_{0}=50 \Omega$. Specifically, equation (5) is interpreted as a voltage-dependent voltage source $0.5 V_{i}$ in series with a currentdependent voltage source $25 I_{i}$ at the analogous ports. The combination of (5) and (6) provides the expression of the physical current as

$$
I_{i}=R_{0}^{-1}\left(A_{i}-B_{i}\right)
$$

which is interpreted as a voltage-dependent current source $0.02 A_{i}$ in parallel with a current-dependent current source $-0.02 B_{i}$ at the physical ports.

The circuit inside multiport $\mathbf{Y}_{S B}$ in Fig. 3 can be synthesized as if $\mathbf{S}$ were an admittance matrix (with units in Siemens), that is, as in Fig. 2. To this purpose, a fictitious branch-admittance matrix whose elements describe the node-to-node admittances is derived from the VF representation of $\mathbf{S}$ in (1) as

$$
\mathbf{Y}_{S B}(s)=\sum_{k=1}^{n} \frac{1}{s-p_{k}} \mathbf{R}_{S B, k}+\mathbf{D}_{S B}+s \mathbf{E}_{S B}
$$

where, in analogy to (2) and (3),

$$
\begin{gathered}
R_{S B, k, i i}=\sum_{j=1}^{N} R_{k, i j}, \quad R_{S B, k, i j}=-R_{k, i j} \\
D_{S B, i i}=\sum_{j=1}^{N} D_{i j}, \quad D_{S B, i j}=-D_{i j} \\
E_{S B, i i}=\sum_{j=1}^{N} E_{i j}, \quad E_{S B, i j}=-E_{i j}
\end{gathered}
$$




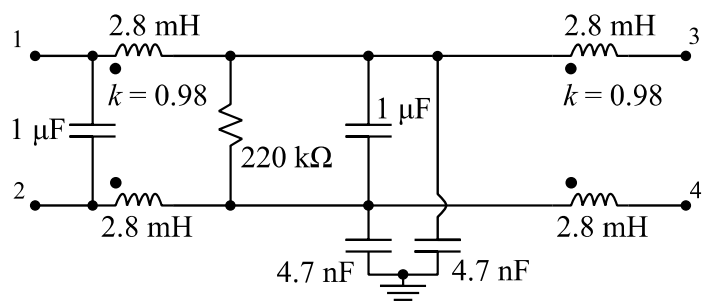

Fig. 4 - Circuit topology and nominal values of parameters declared by the manufacturer of the commercial filter under analysis (except for $k$ ).

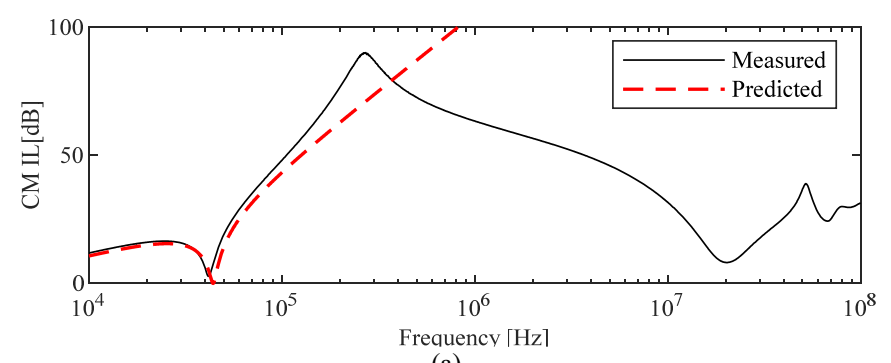

(a)

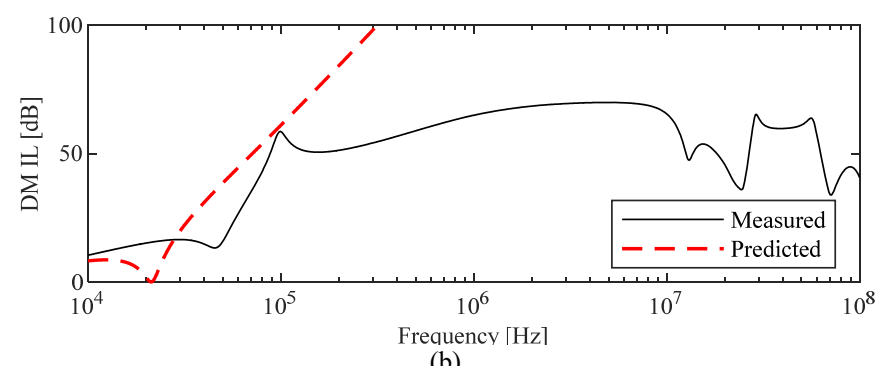

(b)

Fig. 5 - (a) Asymmetrical (CM) and (b) symmetrical (DM) ILs measured according to CISPR 17 (solid lines) are compared to coarse predictions (dashed lines) obtained by the manufacturer's model in Fig. 4. Axes are intentionally limited since predictions become meaningless above few hundreds of $\mathrm{kHz}$.

\section{IMPLEMENTATION EXAMPLE}

In the remainder of this article, a commercial EMI filter with $N_{p}=4$ (model and manufacturer are unessential and undisclosed) is used as a test case to exemplify and validate the proposed modeling approach, as well as to discuss implementation details.

\section{A. EMI Filter Under Analysis}

The EMI filter is sealed inside a metallic enclosure and cannot be internally inspected; therefore, it represents a black box from the viewpoint of modeling. Information provided by the manufacturer on the internal structure is limited to the basic circuit topology in Fig. 4 with ideal components (the coupling coefficient of the mutual inductor is actually not provided, and it is guessed equal to 0.98 ).

The asymmetrical (CM) and symmetrical (DM) ILs measured according to CISPR 17 are shown in Fig. 5. Dashed lines represent predictions obtained by SPICE simulations of the CISPR 17 measurement setups in Fig. 6 involving the basic manufacturer's model in Fig. 4 [11]. Specifically, CM and DM ILs are defined as

$$
I L_{C M}=20 \log _{10}\left|V_{S} \frac{R_{L}}{R_{S}+R_{L}} / V_{L C M}\right|
$$
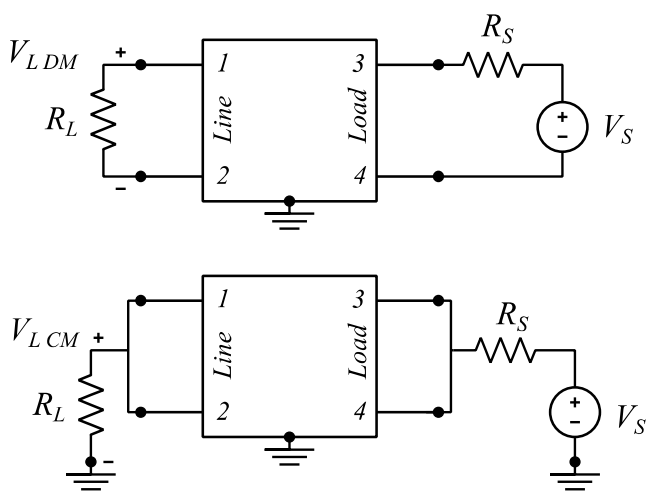

Fig. 6 - Circuits for modeling the CISPR-17 test setups (the central block represents the EMI filter). Top: Symmetrical (DM) IL; Bottom: Asymmetrical (CM) IL.

$$
I L_{D M}=20 \log _{10}\left|V_{S} \frac{R_{L}}{R_{S}+R_{L}} / V_{L D M}\right|
$$

With $R_{L}=R_{S}=50 \Omega$. In Fig. 5, predictions deviate from measurements at very low frequency, after few hundreds of $\mathrm{kHz}$. This example shows that the circuit and component parameters provided in data sheets are of quite limited value for $\mathrm{CE}$ analysis.

\section{B. Setup for S-parameter Characterization}

The use of a metallic enclosure hosting the EMI filter, wires, and coaxial connectors (also recommended in [11] for conventional IL measurement) proved to be fundamental for accurate measurement of the S-parameter matrix. Any measurement error (e.g., coupling of external noise, movement of parts during measurement, etc.) could indeed affect the consistency of the obtained data set. Specifically, corrupted Sparameters would pose insurmountable difficulties in the derivation of a passive macromodel.

The test setup shown in Fig. 7 conforms to such requirements and is composed of a metallic box, whose dimensions do not exceed the dimensions of the EMI filter by more than five centimeters as per [11]. Four bulkhead SMA connectors realize the four measurement ports according to Fig. 1. The filter case is centered and fastened by screws to the metallic box. The center pins of SMA connectors are soldered to pins of suitable terminal blocks, from which wires reach the blade connectors of the filter. It is worth noting that these electrically short interconnections have negligible effect on the frequency

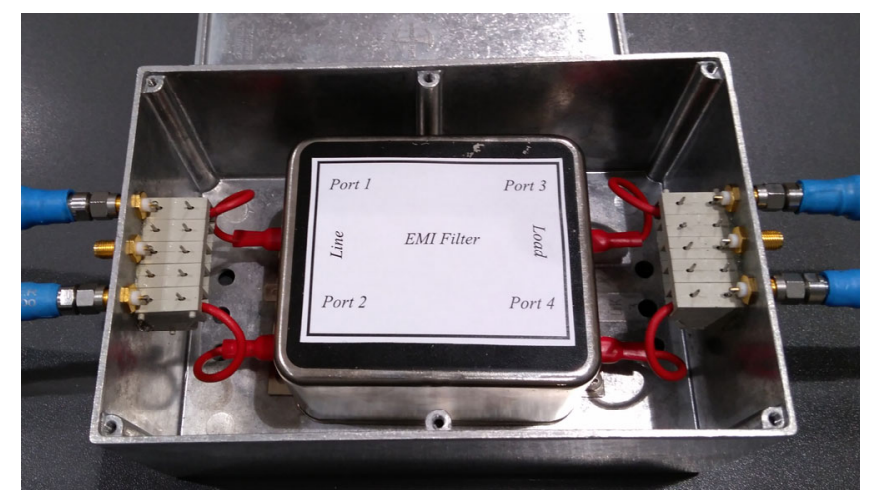

Fig. 7 - Test setup for S-parameter measurement (enclosure is opened) 
response of S-parameters in the band of interest, and therefore they are not de-embedded from measurement results [11].

Measurements were carried out by a Keysight ENA E5071C 4-port VNA in the frequency range $10 \mathrm{kHz}-100 \mathrm{MHz}$, with a narrow intermediate frequency (IF) bandwidth $100 \mathrm{~Hz}$ (which determines a slow sweep time) and 32 averaged sweeps. Once again, the reason for such demanding settings is the need for very accurate VF input data.

\section{Rational Approximation through VF}

To compute a rational approximation (1) of the measured $\mathbf{S}$ matrix by VF, the open-source code vectfit 3 available in [23] based on [16]-[18] was used. For the optimization of the model order, that code was complemented by an iterative routine which, starting from a reasonable small set of poles (e.g., $n=20$ complex-conjugate pairs, logarithmically spaced), increased the number $n$ whenever the VF algorithm did not converge within the desired tolerance (set to 0.005) in a maximum number of iterations (set to 50), [18]. By this approach, the VF algorithm converged with $n=53$ poles ( 23 pairs of complexconjugate poles and 7 real poles).

Three implementation details are worth to be mentioned since they appeared to be necessary for the successful outcome of the whole procedure. First, the underlying theoretical assumption of a reciprocal multiport characterized by a symmetric Sparameter matrix was not perfectly respected by $\mathbf{S}$ owing to unavoidable measurement errors, despite any possible care taken to maximize accuracy. Instead of neglecting the asymmetry (e.g., by processing either the lower or upper triangle of $\mathbf{S}$ ) the best solution was the use of the symmetric part

$$
\mathbf{S}_{\text {sym }}=\frac{1}{2}\left(\mathbf{S}+\mathbf{S}^{\mathrm{T}}\right)
$$

as input to VF, where $\mathrm{T}$ denotes matrix transposition.

The second aspect concerns a suitable choice of the weights of the frequency response [16]-[18], [23]. Weighting is a mean to control the accuracy of VF over the frequency range, by favoring certain elements at the expense of others in the input data set (as far as the error of the least-square solution algorithm is concerned). Among different strategies, the one best suited for EMI filters was found the inverse magnitude

$$
w(s)=1 /\left|S_{s y m, i j}(s)\right|
$$

Indeed, the large dynamic range of the measured S-parameters (almost $80 \mathrm{~dB}$ ) suggests optimization of the relative instead of the absolute error. This is inherently guaranteed by (15) since the smaller the elements, the higher are the associated weights.

Eventually, after the accurate rational expansion (1) was obtained by VF, a final check for unphysical violations of passivity gave positive response. Therefore, results had to be corrected by a passivity-enforcement algorithm based on eigenvalue-perturbation [19], [20].

The amplitude response of measured and fitted S-parameters is plotted in Fig. 8, while the corresponding phase is reported in Fig. 9. The overall fitting is excellent, with limited error only on $\mathrm{S}_{11}$ and $\mathrm{S}_{22}$ around $1 \mathrm{MHz}$.

\section{SPICE circuit model}

An equivalent circuit was synthesized from the rational

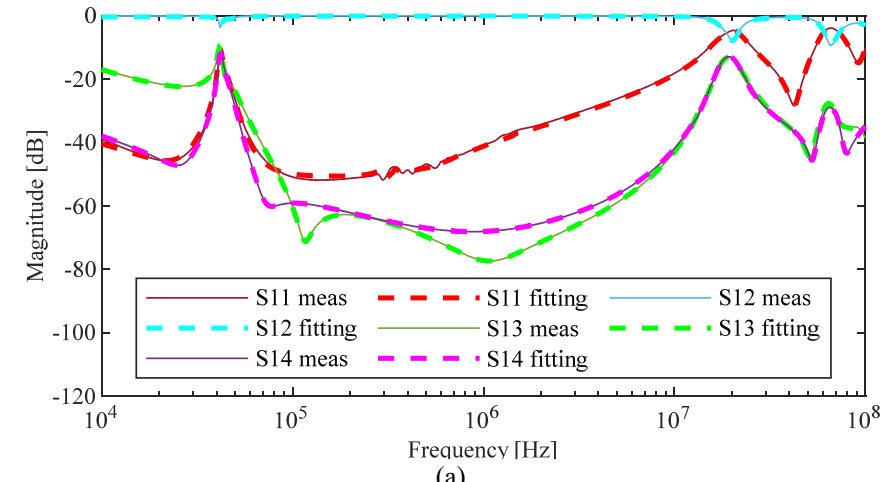

(a)

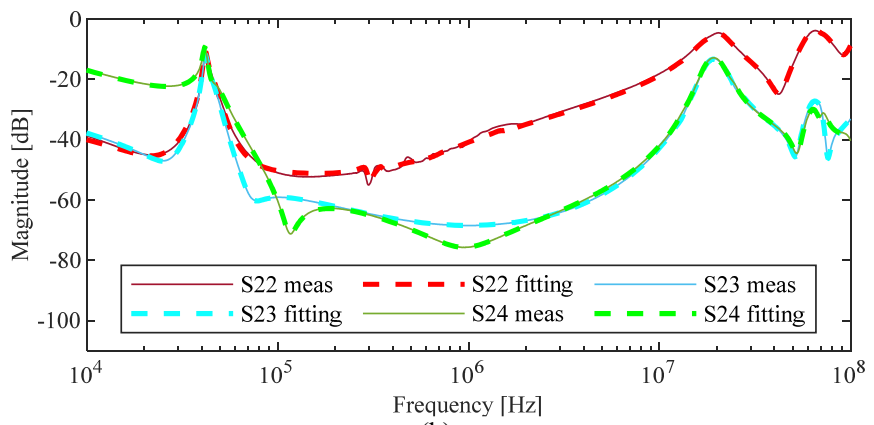

(b)

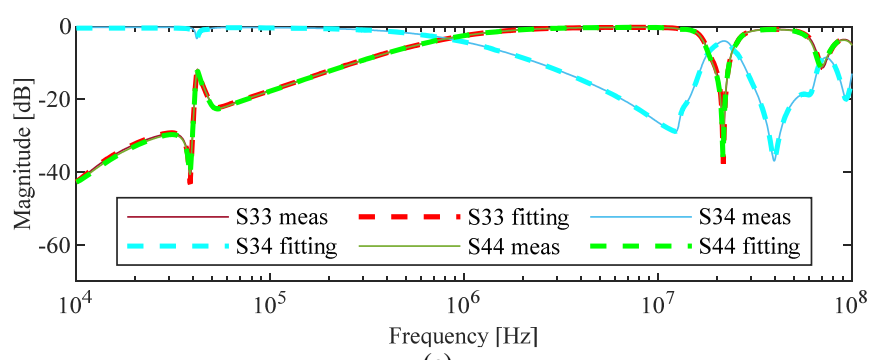

(c)

Fig. 8. S-parameters magnitude: measured (solid lines) vs fitted by rational approximation (dashed lines)

approximation (8) and easily translated into a SPICE netlist. Specifically, each branch admittance in Fig. 2 was realized by the parallel connection of: a) a resistor representing term $D_{S B}$, b) a capacitor for term $s E_{S B}$; c) a resistor and an inductor in series for each real pole in the pole-residue expansion $\left.R_{\mathrm{SB}, k} /\left(s-p_{k}\right) ; \mathrm{d}\right)$ the series connection of a resistor, an inductor, and the parallel between a capacitor and a resistor, for each pair of complex-conjugate poles in that pole-residue expansion [22]. For $N_{p} \times\left(N_{p}+1\right) / 2=10$ branch admittances, 7 real poles, and 23 pairs of complex-conjugate poles, the total number of passive circuit elements in the whole SPICE netlist amounts to 540 resistors, 240 capacitors, and 300 inductors. Additionally, $4 N_{p}=16$ controlled sources complete the circuit according to Fig. 3.

\section{FREQUENCY-DOMAIN SIMULATION OF IL}

The obtained SPICE model can be run in the frequencydomain to predict the IL. To this aim, circuit representations of the CISPR-17 test setups in Fig. 6 are considered, where the center block is the subcircuit containing the whole SPICE model of the filter. 


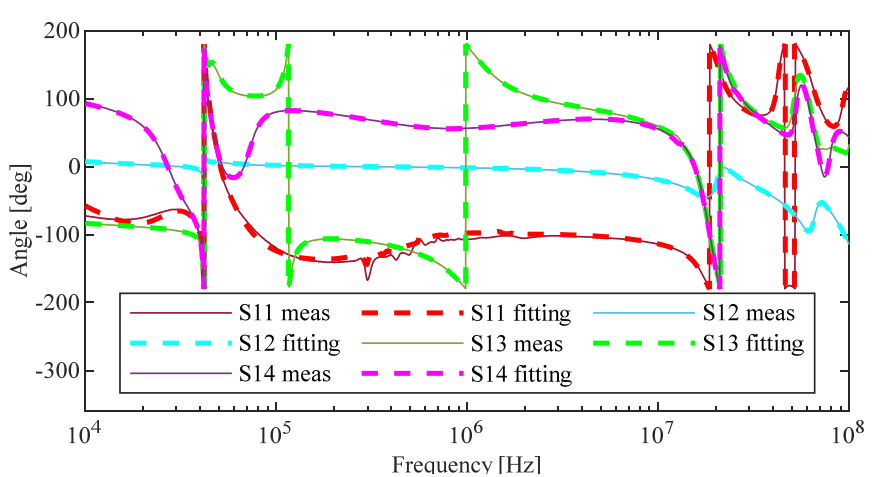

(a)

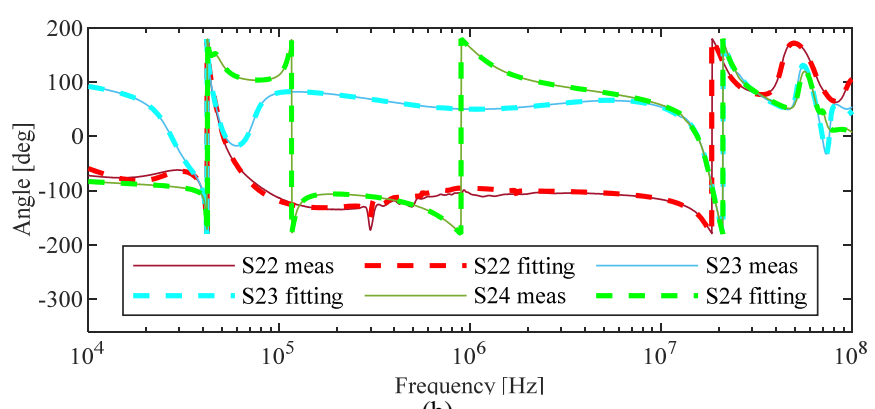

(b)

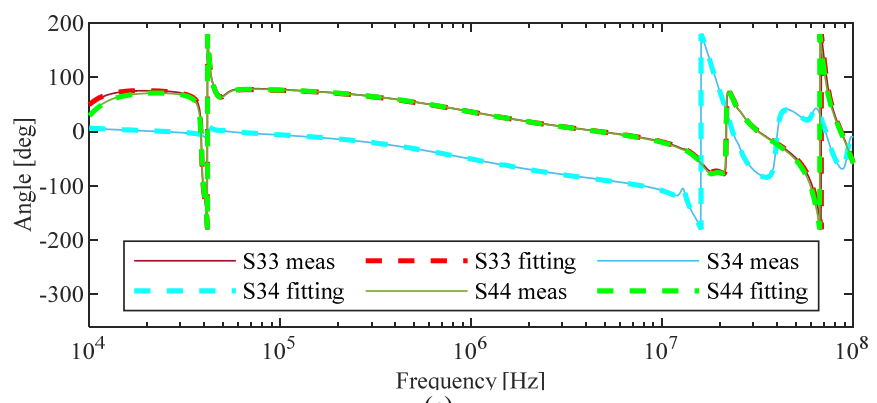

(c)

Fig. 9. S-parameters phase: measured (solid lines) vs fitted by rational approximation (dashed lines)

In Fig. 10, predictions of the standard CM and DM IL (i.e., for $R_{L}=R_{S}=50 \Omega$ ) are plotted and compared versus actual CISPR-17 measurements. The only significant discrepancy (less than $5 \mathrm{~dB}$ ) is observed in a narrow band around $300 \mathrm{kHz}$, where the CM IL gets it maximum value $(90 \mathrm{~dB})$. The origin of this error was carefully investigated and related to the numerical accuracy of the specific SPICE solver [24] in computing small signals at the denominator of the IL ratio, rather than to the rational approximation over which the circuit model is based.

As a second example, predictions of the so-called "0.1-100 $\Omega$ " and "100-0.1 $\Omega$ " DM ILs defined in CISPR 17 Annex C [11] are plotted in Fig. 11. These ILs are intended as extreme figures of merit for non-50 $\Omega$ systems, with the first and second value in their name referred to the source $R_{S}$ and load $R_{L}$ resistance, respectively. Though their definition in [11] is just informative, nowadays most manufacturers use to plot these ILs in data sheets. Their CISPR measurement procedure is challenging since it involves broadband transformers with specific impedance ratios which introduce non-ideal effects (results above few hundreds of $\mathrm{kHz}$ are rarely available indeed). In this respect, the potential of the proposed SPICE model can

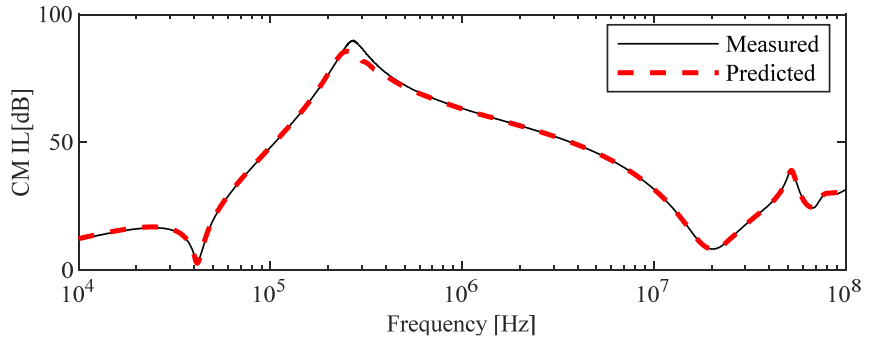

(a)

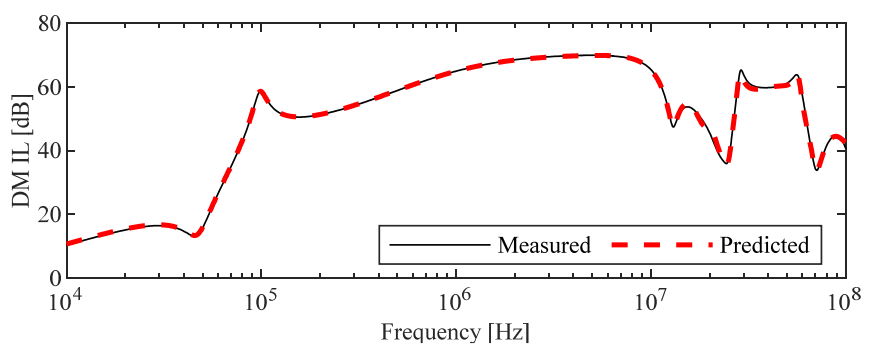

(b)

Fig. 10. (a) Asymmetrical (CM) and (b) symmetrical (DM) ILs measured according to CISPR 17 (solid lines) are compared to predictions (dashed lines) obtained by the SPICE model (frequency-domain simulation).

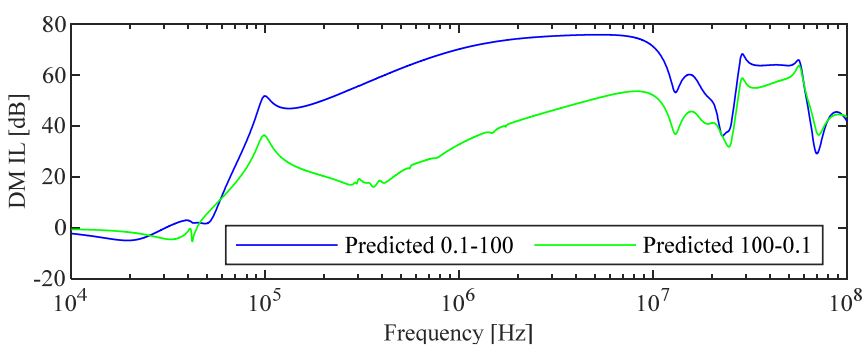

Fig. 11. Prediction of non-50 $\Omega$ symmetrical ILs as defined per CISPR-17 Annex C.

be clearly appreciated, as a mean to evaluate such non-50 $\Omega$ ILs in the whole frequency up to $100 \mathrm{MHz}$ from a general Sparameter characterization of the filter.

In general, a user interested in simulating the IL of the filter could exploit the SPICE circuit model with any load and source impedances (also frequency dependent) better suited to represent the real system/apparatus where the EMI filter under assessment is supposed to operate [3], [5], [15].

\section{Time-domain Simulation of CE}

Time-domain simulation of $\mathrm{CE}$ generated by power electronics converters, which are non-linear and time-variant circuits, really exploits the full potential of the proposed circuitmodeling technique. Indeed, non-linearity of valves and control techniques like pulse-width modulation can be easily described in SPICE and other circuit solvers. The passivity of the subcircuit representing the EMI filter guarantees stability and convergence.

For meaningful time-domain simulations of $\mathrm{CE}$, the frequency range defined to derive the black-box circuit model must include the fundamental harmonic (switching) frequency of the converter. The dc or ac (e.g., 50/60 Hz) spectral components sinthesysed by the converter for power delivery 


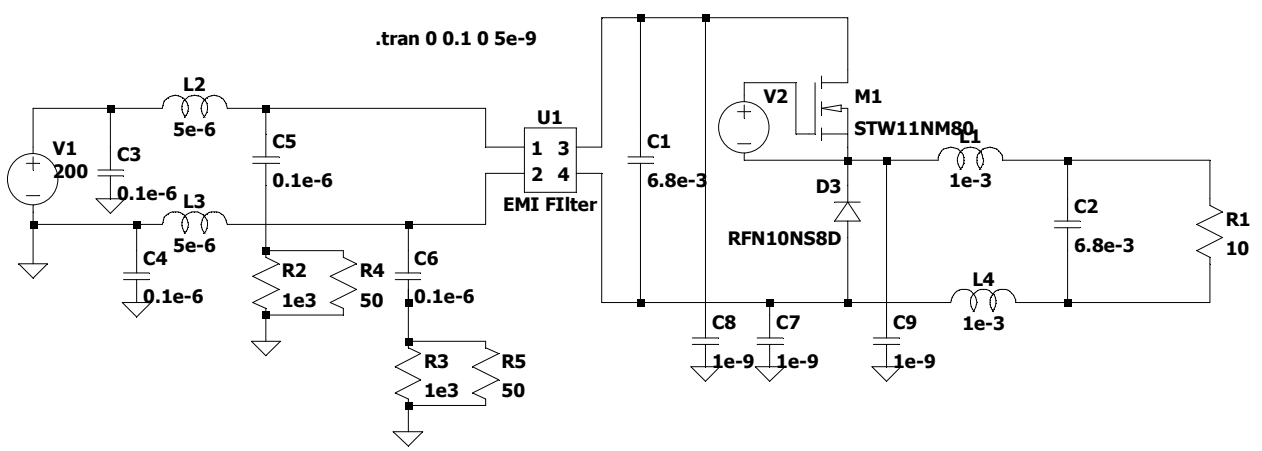

Fig. 12 - SPICE schematic (solver [24]) of the CISPR 25 CE test setup, involving a buck dc/dc converter. The center block represents the subcircuit of the proposed black-box macromodel of the EMI filter.

may be included in that frequency range if accurate prediction of low-frequency quantities (e.g. filter losses, leakage current) were of special interest. This is not even possible in the proposed example, since the lowest frequency is $10 \mathrm{kHz}$ (limited by the VNA). In this case, it is important to check whether the behavior of the equivalent circuit model extrapolated towards dc is compatible with the functional operation of a converter. In this respect, by extrapolating the predicted IL in Fig. 10 for $\omega \rightarrow 0$, one gets $0.2 \mathrm{~dB}$ for the DM, $1.7 \mathrm{~dB}$ for $\mathrm{CM}$, which are small inconsequential values.

To exemplify the inclusion of the black-box filter model in a time-domain simulation procedure [7]-[10], Fig. 12 shows the SPICE schematic of a buck converter in the CISPR-25 [25] test setup. Apart for filter and valves, other system components are ideally modeled (without parasitics) since the illustrative example does not refer to specific equipment. For reliable predictions of CE in specific systems, it would be of paramount importance to develop and include broadband models for the converter, the load, cables or bus bars, accounting for dominant parasitic effects as shown in [8]-[10].

In Fig. 12, the inner block U1 is the subcircuit of the EMI filter. On the right, elements $\mathrm{C} 1$ and $\mathrm{C} 2$ (input and output capacitors), M1 (power mosfet N1STWNM80 by STMicroelectronics), D3 (diode RFN10NS8D by ROHM Semiconductor), L1 and L4 (output inductors) are functional components of the $\mathrm{dc} / \mathrm{dc}$ converter. The voltage source V2 at the gate of M1 drives the valve by a standard PWM switching scheme with a carrier frequency of $10 \mathrm{kHz}$ and a constant 0.75 duty cycle. The resistor $\mathrm{R} 1$ represents a $10 \Omega$ load, which is fed at $150 \mathrm{Vdc}$ and absorbs $2.25 \mathrm{~kW}$. Capacitors C7, C8, C9 establish a parasitic path for CM currents to flow. On the left of the EMI filter, a $200 \mathrm{Vdc}$ voltage source V1 represents the dc

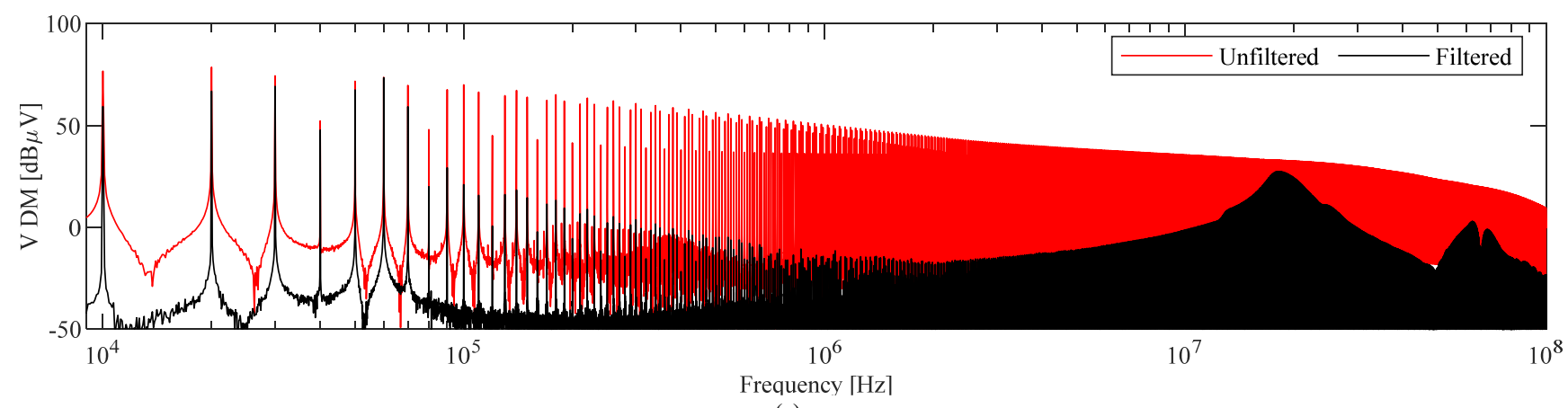

(a)

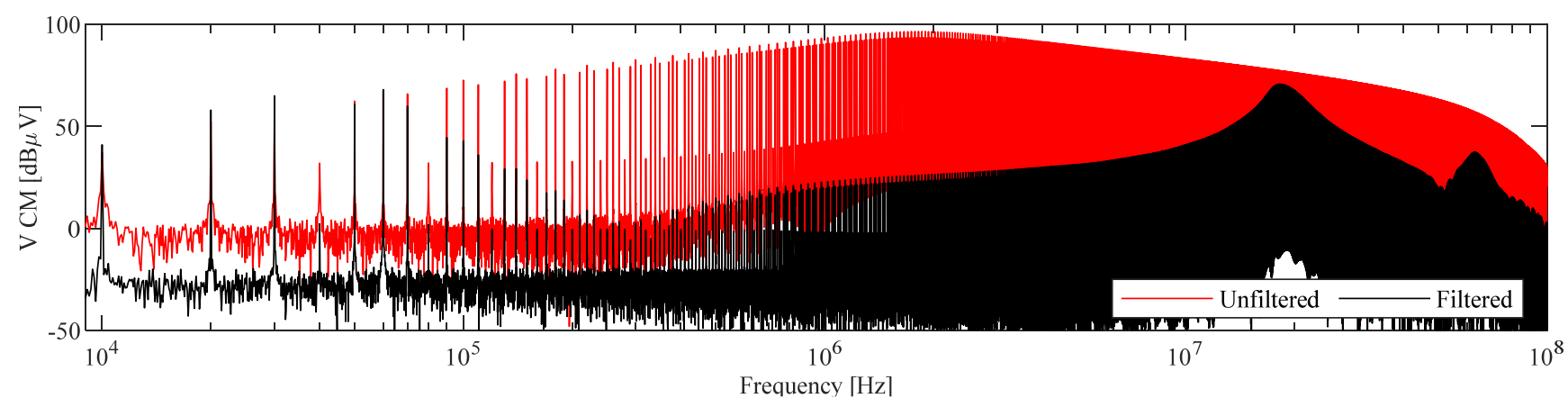

(b)

Fig. 13 - Predictions of (a) DM and (b) CM CE obtained by SPICE time-domain simulation of the CISPR-25 test setup involving a buck converter in Fig. 12 (load $10 \Omega$, absorbed power $2.25 \mathrm{~kW}$ ): without EMI filter (red line); with EMI filter (black line) 


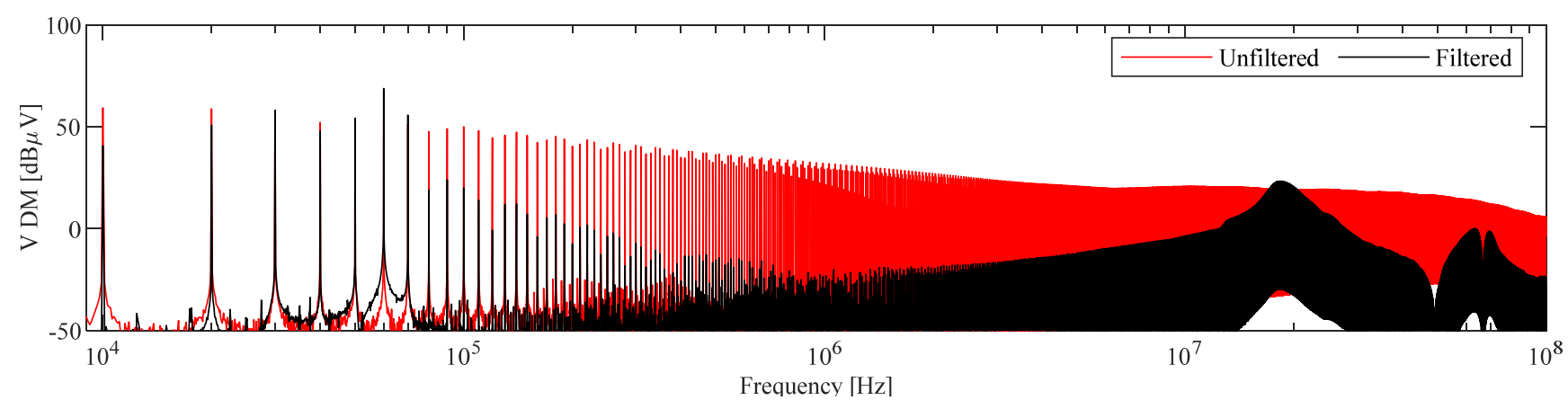

(a)

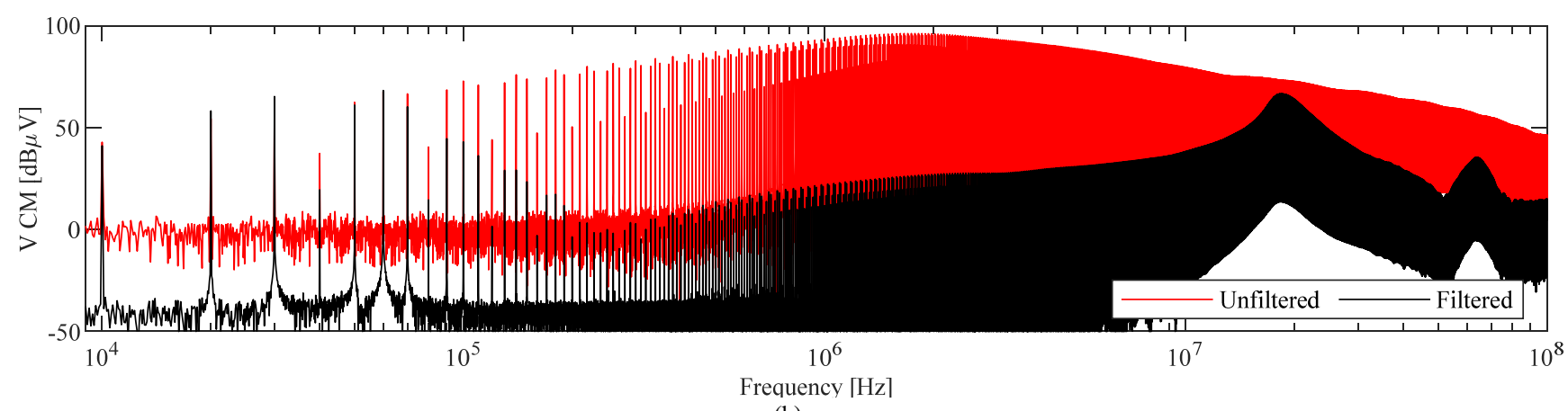

(b)

Fig. 14 - Predictions of (a) DM and (b) CM CE obtained by SPICE time-domain simulation of the CISPR-25 test setup involving a buck converter (load $100 \Omega$, absorbed power $225 \mathrm{~W}$ ): without EMI filter (red line); with EMI filter (black line).

battery, whereas the rest of passive components forms the standard circuit model of the CISPR-25 line-impedance stabilization network (LISN) [25].

$\mathrm{CE}$ are defined as voltages across R4 and R5 for the + and dc wire, respectively, and these waveforms can be directly evaluated by running the SPICE simulation in time domain. The maximum time-step ( $5 \mathrm{~ns}$ ) is consistent with the maximum frequency of interest (100 MHz) according to sampling theory. In the obtained results, the functional start-up transient of the system is discarded (i.e., the first $90 \mathrm{~ms}$ ), so that waveforms are representative of steady-state conditions. Eventually, waveforms are transformed into the frequency domain by the Fast Fourier Transform.

Two SPICE simulations were run: one for the complete circuit in Fig. 12, and another one after removing the EMI filter from the circuit. The obtained spectra of CE are reported in Fig. 13 in terms of CM and DM components of the LISN voltage. A similar set of simulations with and without the filter was carried out for a reduced loading condition, by increasing resistor R1 to $100 \Omega$ (absorbing power $225 \mathrm{~W}$ ), and the obtained CE spectra are plotted in Fig. 14.

For unfiltered CE (red lines), the DM component prevails in the very low frequency range, where single harmonics of the 10 $\mathrm{kHz}$ switching frequency are recognizable. Conversely, the $\mathrm{CM}$ component is dominant above few hundreds of $\mathrm{kHz}$. The comparison of Fig. 13 and Fig. 14 puts in evidence the variability of the spectrum shape with the loading condition of the buck converter, which is sensibly different for CM and DM $\mathrm{CE}$. In terms of $\mathrm{CE}$ levels, the reduction of absorbed power determines a remarkable reduction of DM CE, whereas the $\mathrm{CM}$
CE is less influenced.

The filtered spectra in Fig. 13 and 14 (black line) put in evidence the attenuation of CE made possible by the insertion of the filter. This attenuation results to be largely variable over the frequency range and strictly depending on the operating conditions of the converter in terms of loads. Similarly, any other variation in the operating condition (e.g., different duty cycle, different source voltage, etc.) could be explored by fast circuit simulations. This approach allows the virtual assessment of the performance of the EMI filter in a specific system of interest, which could be hardly inferred by standard frequencydomain ILs reported in data sheets. In this respect, it is worth noting that there may be specific conditions where the insertion of the filter worsens CE levels (see the DM plot in Fig. 14 around $20 \mathrm{MHz}$ ).

\section{CONCLUSION}

The scanty information (nominal circuit and IL) reported in data sheets of EMI filters is generally insufficient to assess the attenuation of $\mathrm{CE}$ through modeling and simulation. On the other hand, manufacturers cannot disclose information on the internal design of their EMI filters to preserve legitimate property rights. To overcome this issue and in order to provide EMC engineers with an effective and accurate tool for the prediction of the filter performance, this paper proposed the derivation of a black-box model (compatible with circuit solvers like SPICE) derived from the S-parameter matrix measured at the external ports of the EMI filter. To this aim, the well-established VF technique for rational approximation of the frequency response of the S-parameters was combined with an original circuit-synthesis approach. 
It was shown that frequency-domain circuit simulation can be used to predict the filter IL for arbitrary load and line impedances. In perspective, this opens the way to the assessment of filter performance through broadband linear models of CE sources and loads [3]-[6]. The full potential of the proposed technique becomes explicit when modeling of EMI filters is to be run in combination with nonlinear, time-variant power-electronic systems, through component-level representations and time-domain circuit simulation [1], [7][10]. An illustrative example (buck converter in a CISPR-25 test setup) was used to highlight the large variability of $\mathrm{CE}$ spectra and EMI attenuation with different operating conditions (output load).

From an industrial viewpoint, the proposed modeling technique seems to represent a step forward in the direction to adopt virtual prototyping, replace lengthy trial-and-error approaches, and better quantify actual filter performance. For instance, if the SPICE netlists were made available by manufacturers of EMI filters as electronic annexes to data sheets, end users would easily be able to assess and select by simulation the optimal model with respect to the specific equipment and CE standard of interest.

\section{REFERENCES}

[1] L. Ran, S. Gokani, J. Clare, K. J. Bradley, and C. Christopoulos, "Conducted electromagnetic emissions in induction motor drive systems part I: Time domain analysis and identification of dominant modes," IEEE Trans. Electromagn. Compat., vol. 13, no. 4, pp. 757-767, Jul. 1998

[2] L. Ran, S. Gokani, J. Clare, K. J. Bradley, and C. Christopoulos, "Conducted electromagnetic emissions in induction motor drive systems part II: Frequency domain models," IEEE Trans. Electromagn. Compat., vol. 13, no. 4, pp. 768-776, Jul. 1998

[3] Q. Liu, F. Wang, and D. Boroyevich, "Modular-Terminal-Behavioral (MTB) model for characterizing switching module conducted EMI generation in converter systems," IEEE Trans. Power Electronics, vol. 21, no. 6, pp. 1804-1814, Nov. 2006.

[4] A. Perez, A. M. Sanchez, J. R. Regue, M. Ribo, P. Rodriguez-Cepeda, F. J. Pajares, "Characterization of power-line filters and electronic equipment for prediction of conducted emissions," IEEE Trans. Electromagn. Compat., vol. 50, no. 3, pp. 77-585, Aug. 2008.

[5] H. M. Rebholz, S. Tenbohlen, and W. Kohler, "Time-domain characterization of rf sources for the design of noise suppression filters," IEEE Trans. Electromagn. Compat., vol. 51, no. 4, pp. 945-952, Nov. 2009

[6] G. Spadacini, F. Grassi, D. Bellan, S. A. Pignari, and F. Marliani, "Prediction of conducted emissions in satellite power buses," Int. Journal Aerospace Engineering, vol. 2015, pp. 1-10, 2015.

[7] G. Spadacini, F. Grassi, and S. A. Pignari, "Modelling and simulation of conducted emissions in the powertrain of electric vehicles," Progress in Electromagn. Res. B, vol. 69, page 1-15, 2016.
[8] M. Laour, R. Tahmi, and C. Vollaire, "Modeling and analysis of conducted and radiated emissions due to common mode current of a Buck Converter," IEEE Trans. Electromagn. Compat., vol. 59, no. 4, pp. 12601267, Aug. 2017.

[9] E. Rondon, F. Morel, C. Vollaire, and J.-L. Schanen, "Modeling of a buck converter with a SiC JFET to predict EMC conducted emissions," IEEE Trans. Pow. Electr., vol. 29, no. 5, pp 2246-2260, May 2014.

[10] Y. Liu, K. Y. See, S. Yin, R. Simanjorang, A. K. Gupta, and J. Lai, "Equivalent circuit model of high power density $\mathrm{SiC}$ converter for common-mode conducted emission prediction and analysis," IEEE Electromagn. Compat. Mag., vol. 8, no. 1, pp. 67-74, 2019.

[11] CISPR 17, Methods of measurement of the suppression characteristics of passive EMC filtering devices, IEC, Jun. 2011.

[12] C. Dominguez-Palacios, P. Gonzalez-Vizuete, M. A. Martin-Prats, and J. Bernal-Mendez, "Smart shielding techniques for common mode chokes in EMI filters," IEEE Trans. Electromagn. Compat., vol. 61, no. 4, pp. 1329-1336, Aug. 2019.

[13] S. Wang, F. C. Lee, and W. G. Odendaal, "Characterization and parasitic extraction of EMI filters using scattering parameters," IEEE Trans. Power Electronics, vol. 20, no. 2, pp. 502-510, 2005.

[14] R. He, Y. Xu, S. Walunj, S. Yong, V. Khilkevich, D. Pommerenke, H. L. Aichele, M. Boettcher, P. Hillenbrand, and A. Klaedtke, "Modeling strategy for EMI filters," IEEE Trans. Electromagn. Compat., vol. 62, no. 4, pp. 1572-1581, Aug. 2020.

[15] M. Haase, K. Hoffmann, and P. Hudec, "General method for characterization of power-line EMI/RFI filters based on S-parameter evaluation," IEEE Trans. Electromagn. Compat., vol. 58, no. 5, pp. $1465-$ 1474, Oct. 2016

[16] B. Gustavsen and A. Semlyen, "Rational approximation of frequency domain responses by Vector Fitting," IEEE Trans. Power Del., vol. 14, no. 3, pp. 1052-1061, July 1999.

[17] B. Gustavsen, "Improving the pole relocating properties of vector fitting," IEEE Trans. Power Del., vol. 21, no. 3, pp. 1587-1592, July 2006.

[18] D. Deschrijver, M. Mrozowski, T. Dhaene, and D. De Zutter, "Macromodeling of multiport systems using a fast implementation of the Vector Fitting method," IEEE Microw. Wireless Compon. Lett., vol. 18, no. 6 , pp. 383-385, June 2008 .

[19] B. Gustavsen and A. Semlyen, "Fast passivity assessment for S-parameter rational models via a half-size test matrix", IEEE Trans. Microw. Theory Techn., vol. 56, no. 12, pp. 2701-2708, December 2008.

[20] B. Gustavsen, "Fast passivity enforcement for S-parameter models by perturbation of residue matrix eigenvalues," IEEE Trans. Adv. Packag., vol. 33, no. 1, pp. 257-265, Feb. 2010.

[21] S. Grivet-Talocia, B. Gustavsen, Passive Macromodeling Theory and Applications, 2016, J. Wiley \& Sons.

[22] G. Antonini, "SPICE equivalent circuits of frequency-domain responses," IEEE Trans. on Electromagn. Compat., vol. 45, no. 3, pp. 502-512, Aug. 2003.

[23] B. Gustavsen, User's guide for vectfit3, Available at: https://www.sintef.no/projectweb/vectorfitting/

[24] Linear Technology Inc., LTspice IV Getting Started Guide, 2011. Available at: https://www.analog.com/en/design-center/design-toolsand-calculators/ltspice-simulator.html

[25] CISPR 25, Vehicles, boats and internal combustion engines - Radio disturbance characteristics - Limits and methods of measurement for the protection of on-board receivers, IEC, Oct. 2016. 